\title{
Time window-size dependence analysis for the minimum semblance approach
}

\author{
D. M. Kamioka (CEP/UNICAMP \& INCT-GP), A. Novais (IMECC/UNICAMP \& INCT-GP), J. Schleicher (IMECC/UNICAMP \& \\ INCT-GP) and H. B. Santos (CEP/UNICAMP \& INCT-GP)
}

Copyright 2016, SBGf - Sociedade Brasileira de Geofísica.

Este texto foi preparado para a apresentação no Simpósio Brasileiro de Geofísica, Ouro Preto, 25 a 27 de outubro de 2016. Seu conteúdo foi revisado pelo Comitê Técnico do VII SimBGf, mas não necessariamente representa a opinião da SBGf ou de seus associados. É proibida a reprodução total ou parcial deste material para propósitos comerciais sem prévia autorização da SBGt.

\section{Abstract}

Minimum Semblance is an alternative approach to determine a quantitative measure for the coherence of seismic events. It is designed to increase the resolution of the semblance sections by using the minimum value of several semblance calculations within a time window. The main advantage of minimum semblance is the resolution improvement along with comparable computational costs to that of conventional function and expressively lower than other coherence measures presented in the literature. Minimum semblance preserves its resolution as the time-window size is increased, in this way becoming less dependent on the choice of the window size than conventional, weighted and $A B$ semblances. To demonstrate this, we vary the inner and outer time window sizes for minimum semblance in applications to synthetic and field data.

\section{Introduction}

Neidell and Taner (1971) introduced semblance as a robust coherence measurement in seismic processing which is especially used to detect events in noisy multiple-coverage data. It supposes white-noise data contamination and constant amplitude along reflection curve. Until now, many attempts have been made to find a more stable measure with less dependence on the type of noise or the choice of parameters used in the analysis.

For instance, weighted semblance (Luo and Hale, 2012) is an immediate extension of the conventional measure for stacking velocity analysis and it uses a weighting function that emphasizes terms that are more sensitive to velocity. $A B$ semblance, introduced by Sarkar et al. 2001 2002 and implemented by Fomel (2009), is interpreted as a correlation measure with an amplitude trend and is notably interesting for data presenting polarity reversal. Minimum semblance as introduced by Kamioka et al. (2015) increases the resolution of the latter while preserving its advantages, including robustness and low computational cost.

In this work we perform numerical tests for minimum semblance using a large range of inner and outer time window sizes through synthetic and field data in an effort to study its behavior. We will see that minimum semblance provides a high resolution even for relatively large time windows, in contrast to other semblance measures, which usually exhibit lower resolution for larger window sizes.

\section{Minimum Semblance}

The conventional coherence measure semblance (Neidell and Taner 1971) is defined as

$$
S=\frac{\sum_{j=-J}^{J}\left(\sum_{i=1}^{I} d_{i, j}\right)^{2}}{I \sum_{j=-J}^{J}\left(\sum_{i=1}^{I} d_{i, j}^{2}\right)},
$$

where $d_{i, j}$ represents the data sample at time index $j$ and trace number $i$. For instance, for a stacking velocity analysis (Taner and Koehler, 1969), $d_{i, j}=d\left(h_{i}, t=T\left(h_{i}\right)+\right.$ $j \Delta t)$, where $T\left(h_{i}\right)=\sqrt{t_{0}^{2}+4 h_{i}^{2} / v^{2}}$, with $v$ denoting the velocity value to be tested at zero-offset time $t_{0}$ and $h_{i}$ the $i$ th half-offset. The inner summation over $i$ corresponds to $I$ traces and the outer summation corresponds to a time window with length $2 J+1$ around the central point at $j=0$.

To define minimum semblance, Kamioka et al. 2015 suggested to use the minimum value of several semblance calculations over a second, outer time window with size $2 K+1$, i.e.,

$$
S_{\min }=\min _{k=-K, \ldots, K} S_{k}
$$

where the semblance values $S_{k}$ are determined inside the inner time window, that is

$$
S_{k}=\frac{\sum_{j=-J}^{J}\left(\sum_{i=1}^{I} d_{i, k+j}\right)^{2}}{I \sum_{j=-J}^{J}\left(\sum_{i=1}^{I} d_{i, k+j}^{2}\right)},
$$

for $k=-K, \ldots, K$. The minimum semblance value $S_{\min }$ is then attributed to the time sample at the central point of the outer time window at $k=0$. They admit a particular case with no inner window at all, i.e., considering the size of the inner window to be a single sample only, or $J=0$. Then, the individual semblance values are calculated as

$$
S_{k}=\frac{\left(\sum_{i=1}^{I} d_{i, k}\right)^{2}}{I\left(\sum_{i=1}^{I} d_{i, k}^{2}\right)},
$$

In this work, we study the behavior of minimum semblance for a range of outer time window sizes with no inner window and compare the results to those of other coherence measure present in literature. Moreover, we investigate the performance of minimum semblance when varying its inner time-window size. 


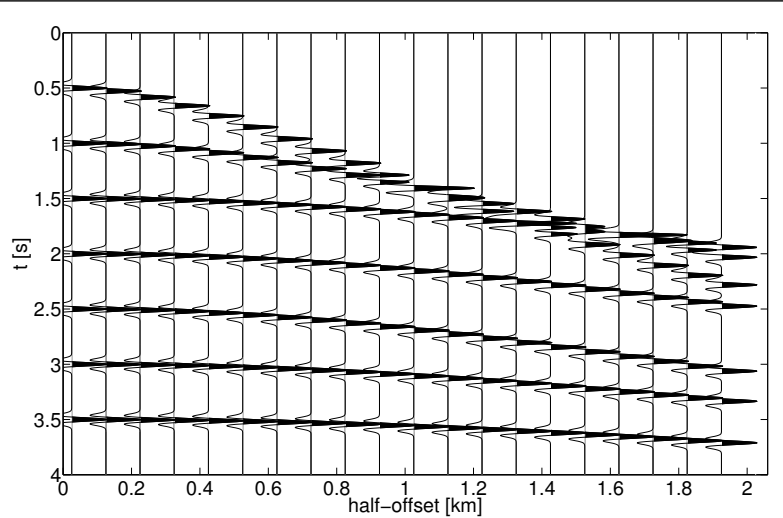

Figure 1: Noise-free synthetic CMP section.

\section{Results}

Synthetic and field data were used to test minimum semblance numerically to investigate the behavior of the semblance measures as a function of the outer and inner time window sizes.

\section{Synthetic data}

A synthetic CMP section is depicted by Figure 1 containing 7 exactly hyperbolic events corresponding to RMS velocities of $1.5,2.0,3.0,2.5,2.0,2.5$ and $3.0 \mathrm{~km} / \mathrm{s}$ at zero-offset times $t_{0}$ of $0.5,1.0,1.5,2.0,2.5,3.0$ and $3.5 \mathrm{~s}$, respectively. Time sampling is $4 \mathrm{~ms}$. To these data, we added random white noise with zero mean at $40 \%$ of the amplitude. We kept the noise the same for the comparison of semblance functions with a given window size. When changing the window size, we replaced it with another realization to obtain a statistically more meaningful result.

Outer window Figures 2,3 and 4 compare the velocity spectra for different window sizes as obtained using minimum semblance without an inner window and with conventional, weighted and $\mathrm{AB}$ semblances. The smallest time window used for the spectra in Figure 2 has the size of 3 samples ( 1 to each side) and the largest time window for Figure 4 has size 21 (10 to each side).

We can note a higher resolution of minimum-semblance sections as compared to the other three semblance measures, independently of the selected time-window size. Moreover, conventional, weighted, and $A B$ semblances lose resolution as the window size increases. In contrast, minimum semblance preserves its resolution behavior for a large range of window sizes. In other words, the results of minimum semblance are much less dependent on the window size. Its resolution stays virtually identical even for rather large time windows. Consequently, the choice of the window size is much less important than for conventional semblance.

High resolution of semblance sections is desirable because the picking process becomes an easier step of seismic processing and the selected velocities can be expected to remain more precise even in the presence of high noise levels. Picking the wrong velocity at this stage may result in incorrect migration and require additional effort in subsequent migration velocity analysis. Thus, if the window size affects the resolution of the semblance spectra

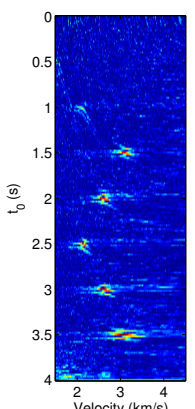

(a)

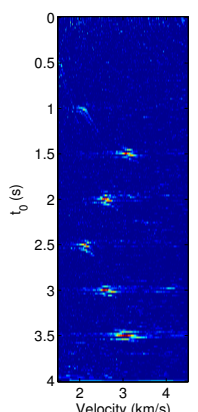

(b)

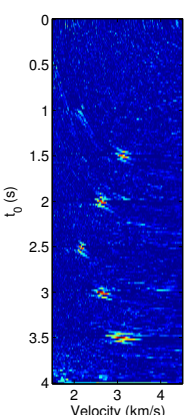

(c)

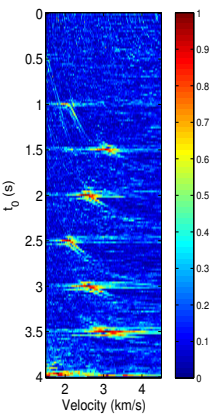

(d)
Figure 2: Velocity spectra for window size 3. Conventional, (b) minimum, (c) weighted,

(d) $A B$ semblance.

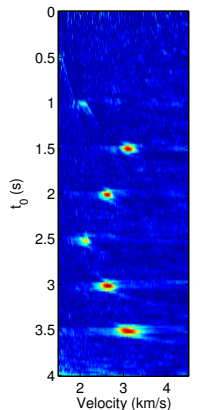

(a)

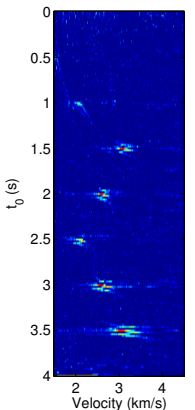

(b)

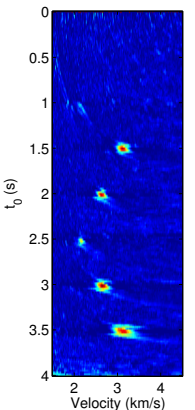

(c)

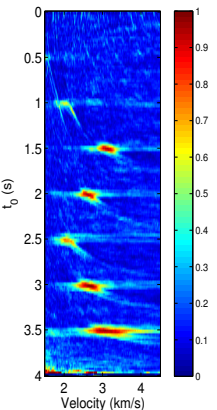

(d)
Figure 3: Velocity spectra for window size $11 . \quad$ (a) Conventional, (b) minimum, (c) weighted, (d) AB semblance.

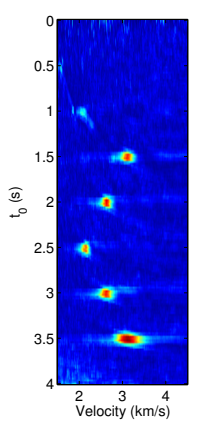

(a)

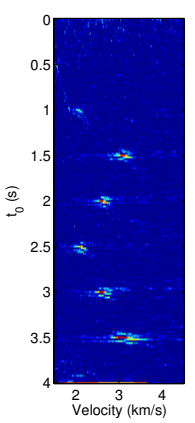

(b)

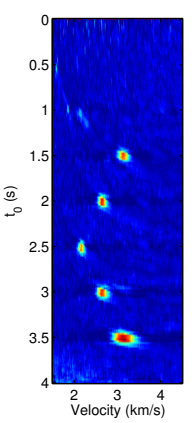

(c)

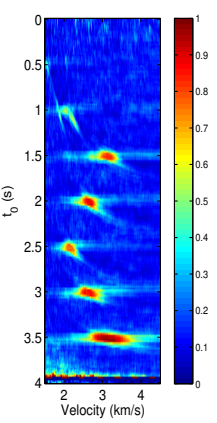

(d)
Figure 4: Velocity spectra for window size 21. (a) Conventional, (b) minimum, (c) weighted, (d) AB semblance.

analyzed, there is a possibility of choosing an inappropriate time window. This possibility is reduced with minimum semblance.

However, an increased resolution might favor a bias in the selected velocities if the position of the semblance peak is incorrect. To investigate whether minimum semblance is subject to this kind of velocity error, we extracted the velocities at the semblance peaks in the spectra of Figures 2 2, 3, and 4 Figures 5 6, and 7 show the resulting errors in velocity and traveltime. We see that the minimum-semblance velocities are very close to the real values of velocities of the synthetic data example. While the velocity errors increase with window size for conventional, weighted and $A B$ semblance, the velocities 


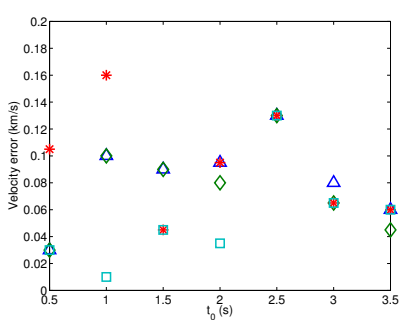

(a)

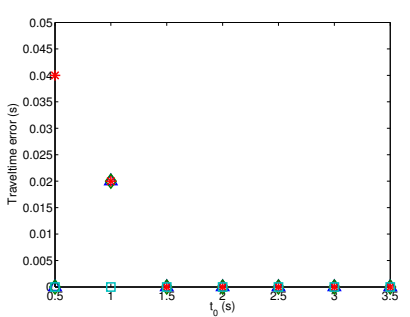

(b)
Figure 5: Parameter error from picking the semblance maxima in Figure 2 using a time window of 3 samples in conventional $(\triangle)$, minimum $(\diamond)$, weighted $(*)$ and $A B$ $(\square)$ semblance. (a) Absolute velocity error. (b) Absolute traveltime error.

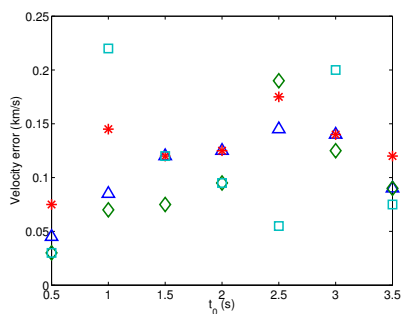

(a)

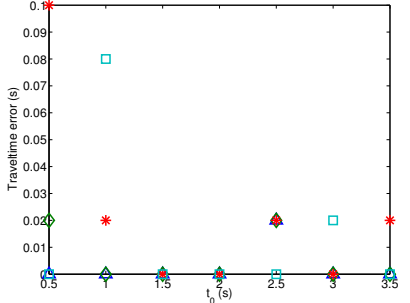

(b)
Figure 6: Parameter error from picking the semblance maxima in Figure 3 using a time window of 11 samples in conventional $(\triangle)$, minimum $(\diamond)$, weighted $(*)$ and $A B$ ( $\square$ ) semblance. (a) Absolute velocity error. (b) Absolute traveltime error.

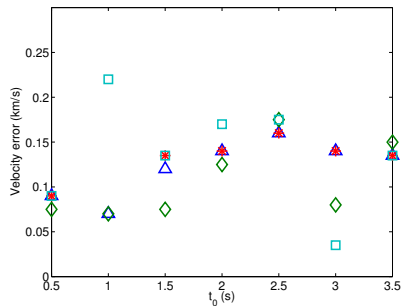

(a)

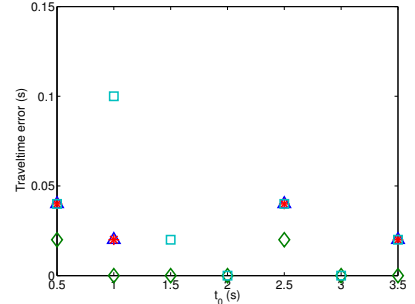

(b)
Figure 7: Parameter error from picking the semblance maxima in Figure 4 using a time window of 21 samples in conventional $(\triangle)$, minimum $(\diamond)$, weighted $(*)$ and $A B$ ( $\square$ ) semblance. (a) Absolute velocity error. (b) Absolute traveltime error.

extracted from the minimum-semblance spectra remain of the same quality. This shows again that velocity-spectra using minimum-semblance are less dependent on the size of the chosen window than using other semblances. Therefore, the optimal window size can be adequately chosen with respect to the noise present in the data, without having to worry about bad velocity picks because of too large or too small windows.

Inner window Figures 8 to 11 exhibit the minimumsemblance velocity spectra for a number of different sizes of the inner and outer time windows. Also shown for comparison are the corresponding velocity spectra for conventional semblance. For the latter, the window size is the same as of the outer minimum-semblance window,

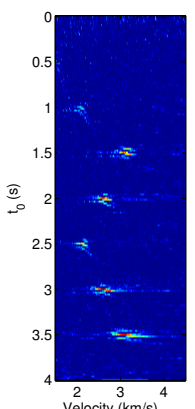

(a)

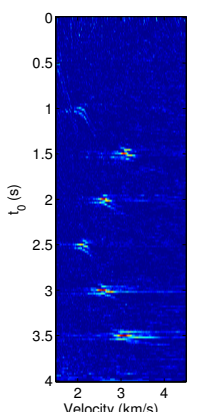

(b)

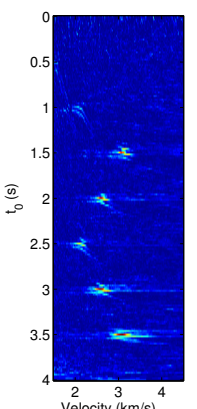

(c)

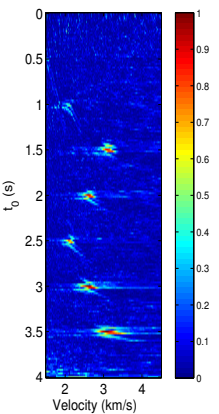

(d)
Figure 8: Velocity spectra with minimum semblance using an outer window of 5 samples (2 to each side), with inner window size (a) 1, (b) 3, and (c) 5 samples. (d) Conventional semblance.

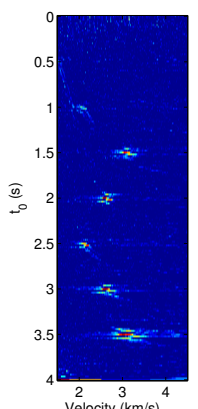

(a)

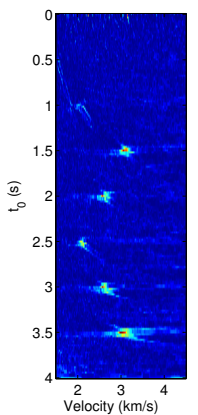

(b)

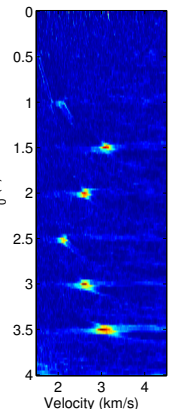

(c)

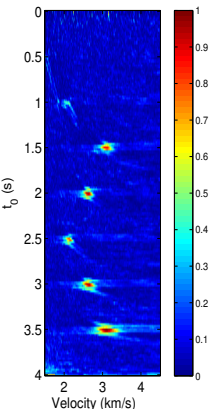

(d)
Figure 9: Velocity spectra with minimum semblance using an outer window of 9 samples (4 to each side), with inner window size (a) 1, (b) 5, and (c) 9 samples. (d) Conventional semblance.

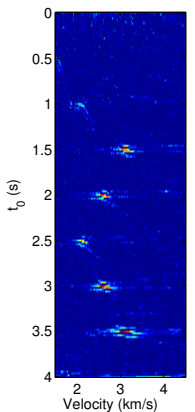

(a)

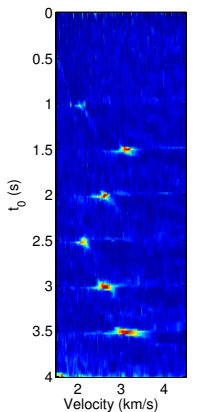

(b)

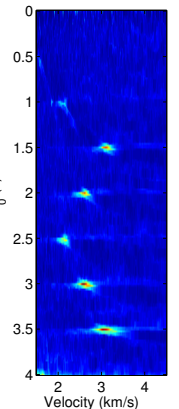

(c)

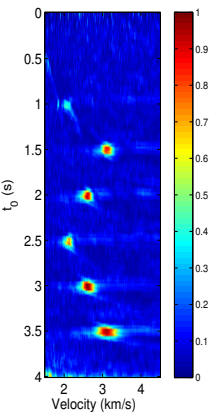

(d)
Figure 10: Velocity spectra with minimum semblance using an outer window of 21 samples (10 to each side), with inner window size (a) 1, (b) 11, and (c) 21 samples. (d) Conventional semblance.

being 5 samples in Figure 8,9 samples in Figure 9, 21 samples in Figure 10, and 41 samples in Figure 11 The inner-window size increases from a single sample in part a of each figure to about half (part b) and full size (part c) of the outer window.

From Figures 8 to 11 we note that minimum semblance without an inner window yields the sharpest peaks, but that the peaks almost disappear for larger windows. The inner window helps to preserve the peaks while still improving resolution over conventional semblance. A choice of an 


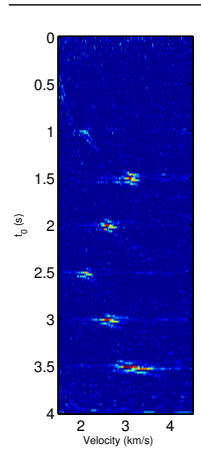

(a)

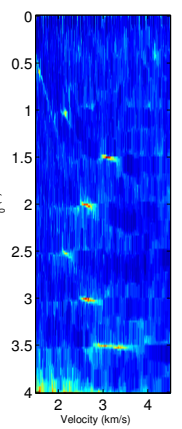

(b)

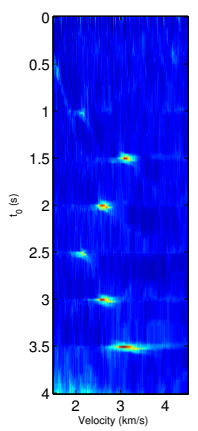

(c)

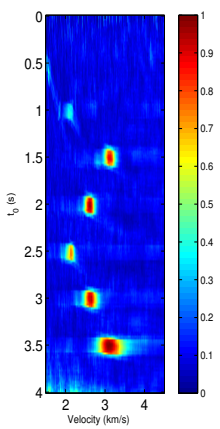

(d)
Figure 11: Velocity spectra with minimum semblance using an outer window of 41 samples (20 to each side), with inner window size (a) 1, (b) 21, and (c) 41 samples. (d) Conventional semblance.

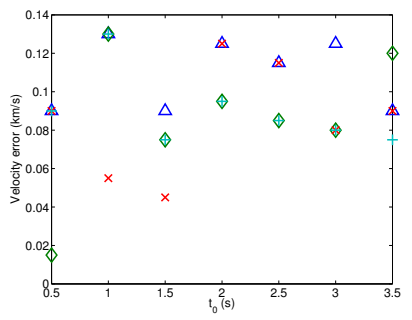

(a)

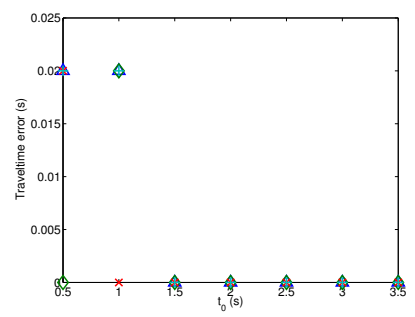

(b)
Figure 12: Parameter error from picking the semblance maxima in Figure 8 using an outer window of 5 samples in conventional semblance $(\triangle)$ and minimum semblance without inner window $(\diamond)$ and with inner windows of $3(\times)$ and 5 samples (+). (a) Absolute velocity error. (b) Absolute traveltime error.

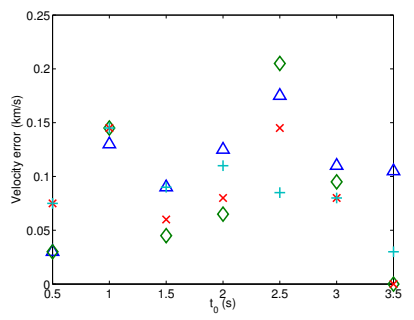

(a)

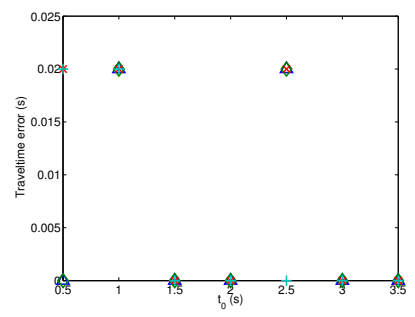

(b)
Figure 13: Parameter error from picking the semblance maxima in Figure 9 using an outer window of 9 samples in conventional semblance $(\triangle)$ and minimum semblance without inner window $(\diamond)$ and with inner windows of $5(\times)$ and 9 samples (+). (a) Absolute velocity error. (b) Absolute traveltime error.

inner window half the size of the outer window seems a good balance between computation cost and resolution without loss of information.

The quality of the parameter extraction as a function of the window size is evaluated in the next set of tests. Figures 12 to 15 show the velocity and normal-traveltime errors generating by picking the semblance maxima in the above velocity spectra.

The velocities are extracted with equivalent precision at a

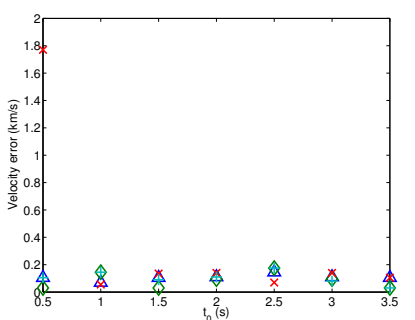

(a)

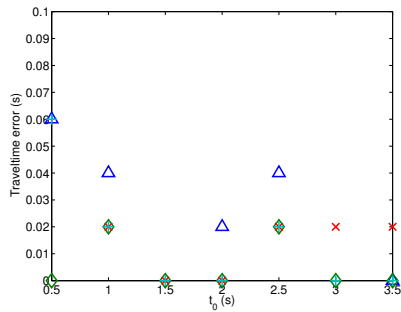

(b)
Figure 14: Parameter error from picking the semblance maxima in Figure 10 using an outer window of 21 samples in conventional semblance $(\triangle)$ and minimum semblance without inner window $(\diamond)$ and with inner windows of 11 $(\times)$ and 21 samples $(+)$. (a) Absolute velocity error. (b) Absolute traveltime error.

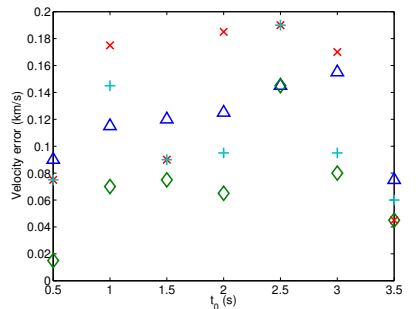

(a)

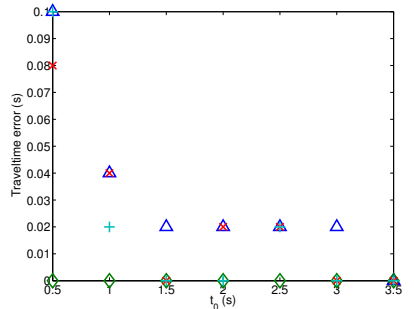

(b)
Figure 15: Parameter error from picking the semblance maxima in Figure 11 using an outer window of 41 samples in conventional semblance $(\triangle)$ and minimum semblance without inner window $(\diamond)$ and with inner windows of 21 $(\times)$ and 41 samples $(+)$. (a) Absolute velocity error. (b) Absolute traveltime error.

comparable error for all window sizes, possibly with a slight advantage for an inner window of half to full size of the outer window. For short lengths of the outer window, minimum semblance without an inner window also provided rather accurate velocity estimates. Larger windows, however, are expected to stabilize the process for larger noise levels.

\section{Field data}

We repeated the above window-size analysis for the fielddata CMP section (Figure 16 of a real marine data set of the Jequitinhonha basin, Brazil. For the real-data tests, no synthetic noise was added to the data. Note that for these tests, we limited $t_{0}$ to the interval from 0 to $4 \mathrm{~s}$ since interpretable reflection events are only present in this time range.

Outer window The tests regarding the outer timewindow on the field data confirmed the results obtained with the synthetic data above (cf. Figures 17, 18 and 19. As before, we see that the resolution of minimumsemblance velocity spectra is higher than that of other semblance measures, irrespectively of the chosen size of the outer time window. Minimum semblance results show less dependency on the outer time window size than the other measures. Also, its resolution is preserved even for rather large time windows, which makes the choice of the window size less important than for conventional, weighted, 


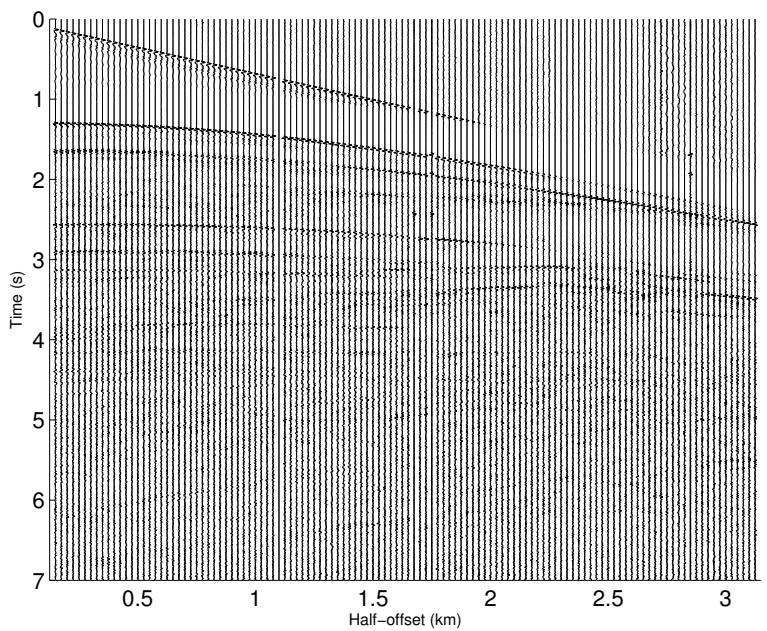

Figure 16: CMP section for field data.

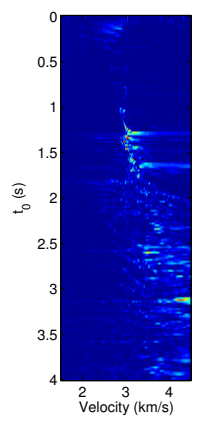

(a)

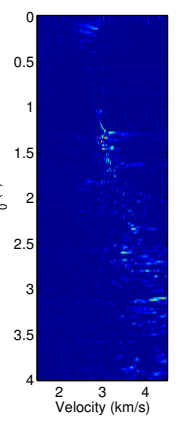

(b)

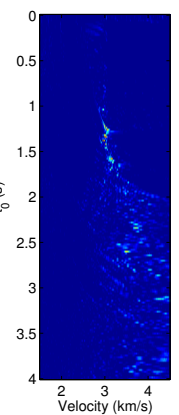

(c)

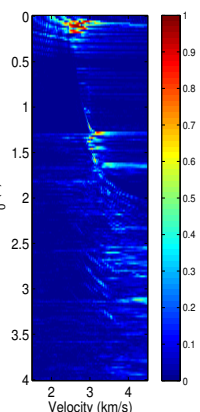

(d)
Figure 17: Velocity spectra for outer window size 3 . (a) Conventional, (b) minimum, (c) weighted, (d) $A B$ semblance.

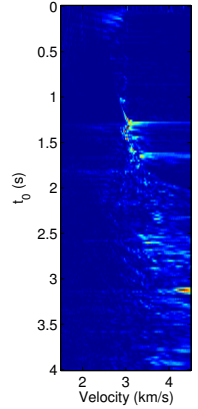

(a)

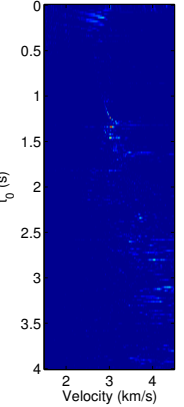

(b)

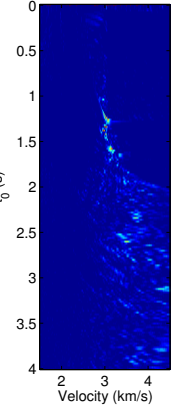

(c)

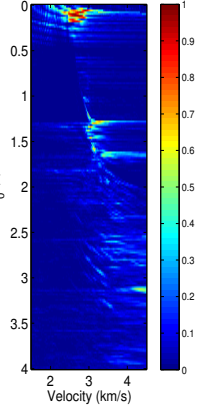

(d)
Figure 18: Velocity spectra for outer window size 5 (a) Conventional, (b) minimum, (c) weighted, (d) $A B$ semblance.

and $A B$ semblances. Note, however, that if the window is larger than the seismic wavelet, minimum semblance will no longer detect the events.

Inner window We analyzed the performance of minimum semblance with different outer window sizes from 5 to 21 samples and inner window sizes from 3 samples to full outer window size. Figures 20 to 25 depict the velocity spectra, comparing the best results reached by minimum semblance to those of conventional semblance. Note that minimum semblance yields higher resolution in the

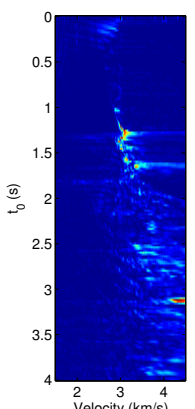

(a)

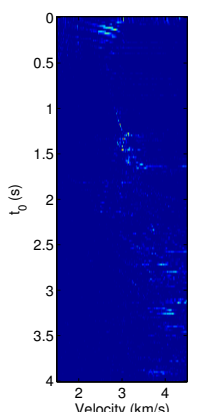

(b)

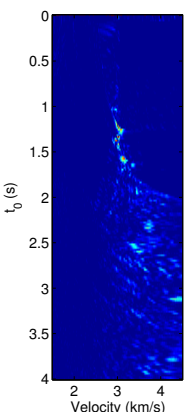

(c)

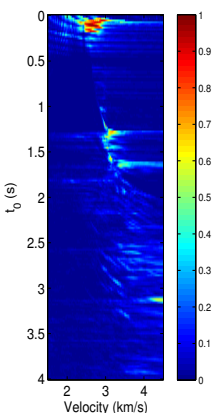

(d)
Figure 19: Velocity spectra for outer window size 7. (a) Conventional, (b) minimum, (c) weighted, (d) $A B$ semblance.

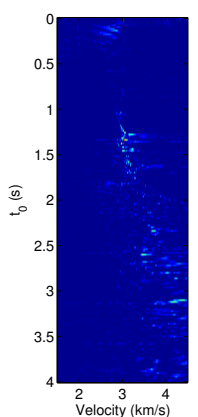

(a)

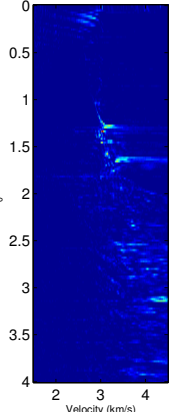

(b)

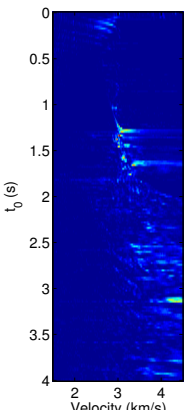

(c)

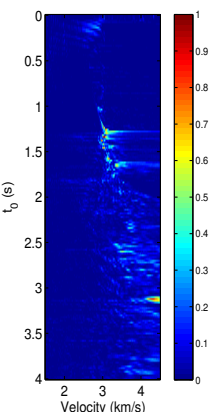

(d)
Figure 20: Velocity spectra with an outer window of 5 samples, inner window size (a) 1 , (b) 3 , and (c) 5 samples. (d) Conventional semblance.

corresponding spectra, and the visualization of the velocity trend on the semblance panel is somewhat better than the conventional approach when choosing an inner window about half the size of the outer window or slightly larger. This observation is again in agreement with the results for the synthetic data. The velocity trend is probably best noticeable in Figure 21 , which was obtained with an outer window of 7 samples and an inner one of 5 samples, in Figure 22p with outer window of 9 samples and inner one of 5 samples, or in Figure 23, with outer window of 11 samples and inner one of 7 samples. For larger inner windows, the minimum-semblance spectra begin to exhibit the same out-of-focus aspect as the conventionalsemblance spectra, and for larger outer windows, the quality of the velocity spectra begins to deteriorate.

\section{Conclusions}

In this work, we have investigated the dependence of a recently proposed coherence measure called minimum semblance on the size of the involved time windows. We have seen that velocity spectra calculated using minimum semblance rather than other semblance measures such as conventional, weighted or $A B$ semblances, exhibit increased resolution when used with comparable window size. In addition to the outer window, minimum semblances allows for the use of an inner window that can help to stabilize results. When an inner window is used, minimum semblance still provides higher resolution as compared to the other measures. If used without an inner window, minimum semblance has the same computational cost as 


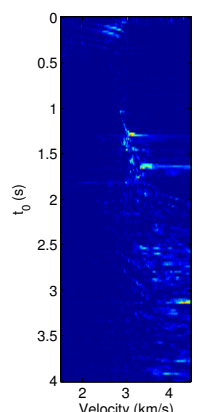

(a)

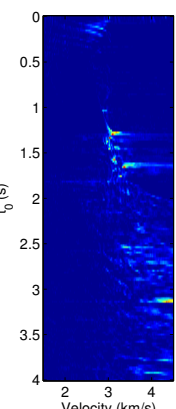

(b)

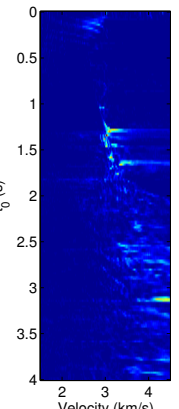

(c)

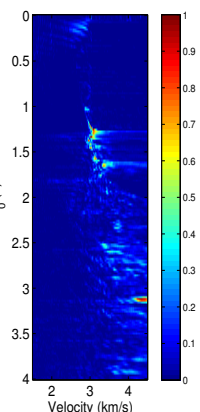

(d)
Figure 21: Velocity spectra with an outer window of 7 samples, inner window size (a) 3, (b) 5 , and (c) 7 samples. (d) Conventional semblance.

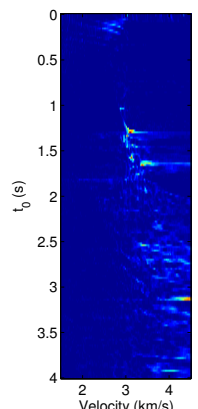

(a)

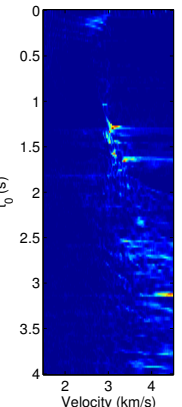

(b)

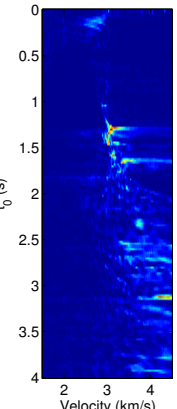

(c)

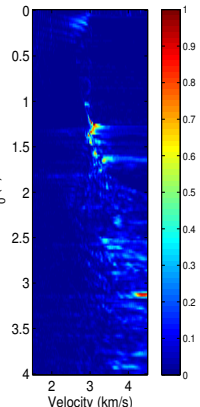

(d)
Figure 22: Velocity spectra with an outer window of 9 samples, inner window size (a) 3, (b) 7 , and (c) 9 samples. (d) Conventional semblance.

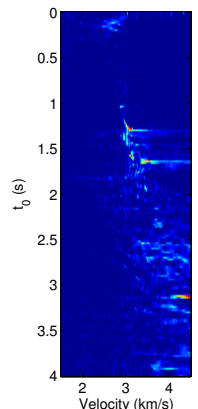

(a)

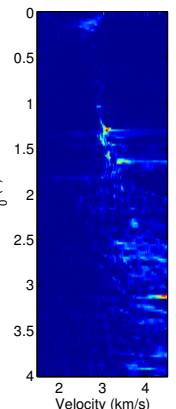

(b)

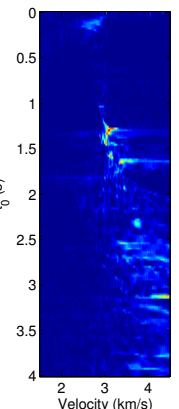

(c)

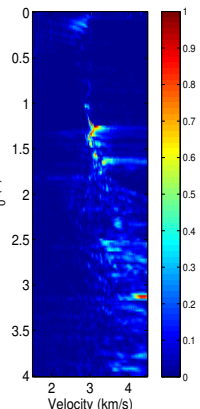

(d)
Figure 23: Velocity spectra with an outer window of 11 samples, inner window size (a) 5, (b) 7, and (c) 9 samples. (d) Conventional semblance.

conventional semblance. The use of an inner window slightly adds to the computational cost. Care has to be taken not to choose the outer window larger than the seismic wavelet, because in that case minimum semblance will no longer detect the events. Our numerical tests indicate that the visualization of the velocity trend on the semblance panel is improved when choosing an inner window approximately half the size of the outer window or slightly larger. Overall minimum semblance is virtually independent of the time-window size. This is a relevant benefit over the other tested measures, the results of which strongly depend on the choice of the window size.

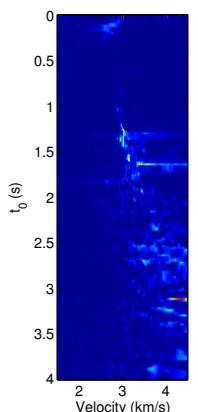

(a)

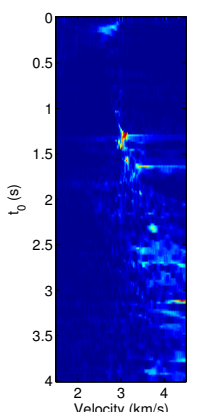

(b)

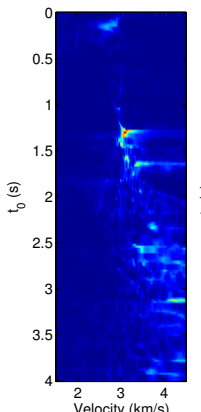

(c)

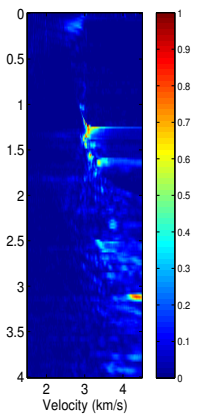

(d)
Figure 24: Velocity spectra with an outer window of 15 samples, inner window size (a) $5,($ (b) 9 , and (c) 15 samples. (d) Conventional semblance.

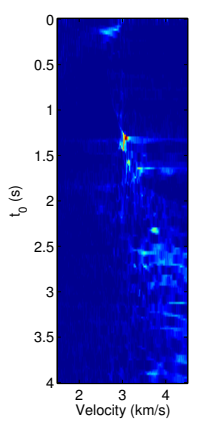

(a)

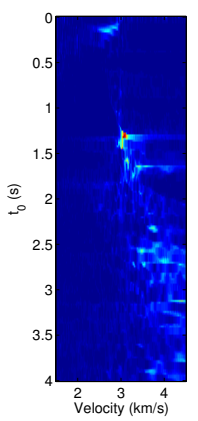

(b)

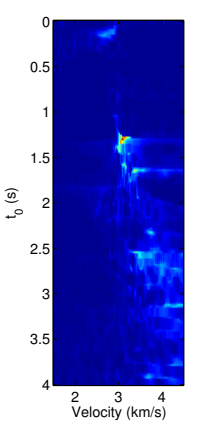

(c)

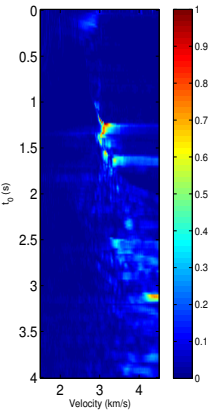

(d)
Figure 25: Velocity spectra with an outer window of 21 samples, inner window size (a) 9, (b) 13, and (c) 19 samples. (d) Conventional semblance.

\section{Acknowledgments}

We thank PRH-PB 230, ANP, PETROBRAS, CAPES, $\mathrm{CNPq}$, and the sponsors of the Wave Inversion Technology (WIT) Consortium for financial support.

\section{References}

Fomel, S., 2009, Velocity analysis using $A B$ semblance: Geophysical Prospecting, 57, 311-321, doi: 10.1111/j.1365-2478.2008.00741.x.

Kamioka, D., A. Camargo, A. Novais, J. Schleicher, and L. Santos, 2015, Minimum semblance: A new coherence measure to improve resolution of semblance sections: Fourteenth International Congress of The Brazilian Geophysical Society, 1316-1320, doi: 10.1190/sbgf2015-261.

Luo, S., and D. Hale, 2012, Velocity analysis using weighted semblance: Geophysics, 77, U15-U22, doi: 10.1190/geo2011-0034.1.

Neidell, N. S., and M. T. Taner, 1971, Semblance and other coherency measures for multichannel data: Geophysics, 36, 482-497, doi: 10.1190/1.1440953.

Sarkar, D., R. Baumel, and K. Larner, 2002, Velocity analysis in the presence of amplitude variation: Geophysics, 67, 1664-1672, doi: 10.1190/1.1512814.

Sarkar, D., J. P. Castagna, and L. W. J., 2001, AVO and velocity analysis: Geophysics, 66, 1284-1293, doi: 10.1190/1.1487076.

Taner, M. T., and F. Koehler, 1969, Velocity spectra - digital computer derivation and applications of velocity functions: Geophysics, 34, 859-81, doi: 10.1190/1.1440058. 